\title{
Le soin, vu par la philosophie, les humanités, la sociologie
}

\section{Jean Martin}

Dr med., membre de la rédaction

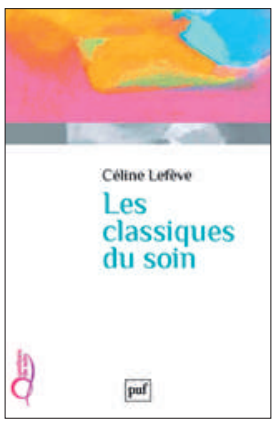

Céline Lefève, Lazare Benaroyo, Frédéric Worms (dir. publ.) Les classiques du soin

Paris: Presses universitaires de France (PUF); 2015. 228 pages. $20.10 \mathrm{CHF}$. ISBN 978-2-13072988-4
Sous une forme ramassée, c'est une anthologie choisie de textes traitant de la relation soigné-soignant. Les travaux de dix-huit auteurs majeurs sont présentés et débattus par quatorze académiques (quatre d'entre eux rédigeant deux contributions). Selon les directeurs de publication: «Ces textes ne traitent pas de la médecine comme d'un champ technique particulier mais du soin comme nécessité indissociablement vitale et morale. L'enjeu étant de permettre au malade de (re)devenir, si possible, l'acteur du rétablissement de son identité altérée par la maladie [...] Ces textes disent les expériences vécues de la maladie ainsi que l'imbrication des sciences et des pratiques, des faits et des valeurs.» Si le champ d'étude est les soins de santé, c'est au plan philosophique, et plus avant socio-anthropologique et par la littérature surtout. Il est fait référence à l'émergence depuis les années 1990 de la médecine narrative et au retour des humanités dans les études de médecine.

Dans la première partie, «Le soin chez les classiques», les auteurs étudiés vont de Hippocrate et Aristote à Montaigne et John Locke puis à Tolstoï, entre autres. La deuxième, "Les réflexions fondatrices», présente des grands incontournables: Balint, Canguilhem, Levinas, Ricœur et Jonas, ainsi que Fritz Zorn, le Zurichois de bonne famille et son livre Mars. Dans la troisième, "Classiques d'aujourd'hui», sont discutés les travaux de Michel Foucault, de l'anthropologue hollandaise Annemarie Mol et de quatre auteurs anglo-saxons (Goffman, Strauss, Sacks et Sontag).

En quelque 200 pages de petit format, il ne peut être question de faire le tour de ce qu'il faudrait recommander. «Les textes choisis ici reflètent les transformations des conceptions et pratiques. Dès la Grèce antique, on voit apparaître la tension critique entre l'autonomisation du soin et le souci du sujet malade [...] Autre tension qui va croissant, celle entre le devenir scientifique de la médecine et l'occultation de la subjectivité du malade.» Ce qui peut être illustré par cette phrase de Georges Canguilhem: «La prise en charge d'un malade ne relève pas de la même responsabilité que la lutte rationnelle contre la maladie.»

\section{Canguilhem, Ricœur}

Céline Lefève relève que Canguilhem réalise bien que les soins médicaux se jouent non dans une sorte d'enclos réservé mais situés en un lieu et une époque donnés: «la conscience que les malades ont de leur situation n'est jamais nue, sauvage»; elle est construite par l'histoire et la société. Elle prend aujourd'hui des formes contradictoires: la confiance dans l'efficacité thérapeutique coexiste avec la critique des effets iatrogènes, l'exigence croissante de santé et de sécurité publiques coexiste avec la revendication d'autonomie.

Didier Sicard, discutant l'apport de Paul Ricœur: «Du côté du médecin, le pacte de soins repose sur l'engagement à diagnostiquer, à agir selon les données actualisées et à ne pas abandonner son malade. Or le pacte de soins est actuellement en voie d'éclatement, brisé parce que la méfiance est encouragée au détriment de la confiance» - faisant référence notamment à la présence croissante d'Internet dans la relation. Oui, mais (note de J. M.) tout indique qu'Internet ne s'évanouira pas comme un mauvais rêve et qu'il s'agit de composer avec lui, limiter les risques tout en optimisant les possibles bénéfices.

\section{Deux Américains}

Erving Goffman est un sociologue qui s'est intéressé aux populations hébergées ou enfermées dans des institutions (hôpitaux, internats, prisons, casernes). Il constate une destinée semblable pour l'ensemble des reclus - un "caractère commun au remodelage que subissent les personnes une fois admises à l'hôpital, remodelage social qui peut affecter les humains les plus irréductiblement hétéroclites». Dans le même sens, Ricœur a insisté sur le fait que la souffrance et la médecine tendent à faire régresser l'estime de soi du malade.

Anselm Strauss, autre sociologue américain, a élaboré la notion de trajectoire de maladie, qui inclut toute l'organisation du travail déployée autour du patient. A ce propos: «Malgré la place prépondérante des rou- 
tines en médecine, une réflexivité des équipes sur leur fonctionnement quotidien est possible. Les soignants peuvent agir sur le cadre structurant leurs actions. Cela ouvre la voie à la réflexion dans ses aspects éthiques, en prenant du recul devant tels évènements ou cas complexes.» Ces problèmes demandent de discuter des décisions d'abstention ou d'arrêt de traitement, questions posées en fin de vie notamment.

\section{Non à l'homo economicus}

Les travaux de Annemarie Mol, d'Amsterdam, la plus contemporaine des auteurs présentés, retiennent particulièrement l'attention en niant la pertinence, dans les soins, de l'homo economicus - à savoir du modèle du patient rationnel qui se détermine en fonction d'éléments objectifs. Le malade, dit-elle, n'est pas en premier lieu un "sujet qui choisit» mais plutôt un sujet "en actes» (plus qu'il ne fait des choix, il est amené à agir afin de s'ajuster aux aléas de la maladie et être soigné et soutenu). Elle souligne l'importance des échanges entre soigné et soignant "Comment ajuster les uns aux autres de la meilleure façon possible tous les éléments qui sont concernés dans la vie quotidienne d'un patient.» Dans la pratique, "les décisions ne correspondent pas simplement à une direction donnée aux patients par les soignants, ni l'inverse, mais à un bricolage partagé.

\section{Question de sens}

Dans ses livres connus (La maladie comme métaphore, Le sida et ses métaphores), l'auteure américaine Susan Sontag insiste sur le fait qu'il ne s'agit pas de chercher ou de vouloir donner du sens à la maladie: «Considérer le cancer comme juste une maladie - certes très grave, mais juste une maladie. Ni une malédiction, ni une punition, ni une honte. Une entité dépourvue de sens». Point majeur, entrant en contradiction avec notre compatriote Fritz Zorn parlant dans Mars de son expérience de malade du cancer, accordant beaucoup de signification à ce qui lui arrive: "Je descends d'une des meilleures familles de Zurich. J'ai eu une éducation bourgeoise. Ma famille est passablement dégénérée et je suis abîmé par mon milieu. Naturellement, j’ai aussi le cancer [...] D'une part, c'est une maladie du corps; d'autre part, c'est une maladie de l'âme dont je ne puis dire qu'une chose: c'est une chance qu'elle se soit enfin déclarée.»

\section{Besoin d'un regard large}

Dans la partie «Réflexions fondatrices», les apports discutés sont d'Europe continentale. La troisième partie elle présente surtout des auteurs anglo-saxons; ses textes, relève-t-on, «battent en brèche la figure, héritée notamment d'une tradition paternaliste, d'un patient dont le statut et l'existence seraient intégralement définis par le système médical.» Elle a aussi le mérite d'apporter un regard incluant des aspects de santé publique et sociologiques, élargissant l'angle de vue auparavant focalisé sur le colloque singulier. On y relève aussi, de façon à mon sens nécessaire, une dimension de contingence, de relativité des circonstances, des choses et des gens et, partant, des déterminations pragmatiques (position qui n'a pas toujours bonne presse en éthique médicale, notamment en milieu francophone). Or, nombre de situations dans l'activité de soins admettent des positions, réflexions et réponses diverses (il n'y pas qu'une seule bonne solution, suivant tel principe absolu...).

Les classiques du soin n'est pas un manuel qui, avec un objectif didactique précis, voudrait dire tout (ou l'essentiel de) ce qu'on doit savoir du rapport soigné-soignant, au plan éthico-psycho-social et dans une optique pratique. C'est plutôt la présentation par des experts à un titre ou l'autre de sciences humaines - philosophes mais aussi quatre médecins - d'œuvres majeures sur le sujet. En conclusion, je citerai la pertinente formulation des directeurs de publication: "On n'apprend pas les classiques, on les ouvre, on les comprend, on les discute, on les critique aussi. Ce volume ne vise pas à dégager un sens univoque du soin, ni un accord entre ces textes, mais à éclairer les discussions les plus actuelles.» 